\title{
BMJ Open SystEmatic review and meta-aNAlysis of infanT and young child feeding Practices (ENAT-P) in Ethiopia: protocol
}

\author{
Tesfa Dejenie Habtewold, ${ }^{1,2}$ Md. Atiqul Islam, ${ }^{2}$ Nigussie Tadesse Sharew, ${ }^{1}$ \\ Shimels Hussien Mohammed, ${ }^{3}$ Mulugeta Molla Birhanu, ${ }^{4}$ \\ Balewgizie Sileshi Tegegne ${ }^{4}$
}

To cite: Habtewold TD, Islam MA, Sharew NT, et al. SystEmatic review and metaaNAlysis of infanT and young child feeding Practices (ENAT-P) in Ethiopia: protocol. BMJ Open 2017;7:e017437. doi:10.1136/ bmjopen-2017-017437

- Prepublication history and additional material are available. To view these files please visit the journal online (http://dx.doi org/10.1136/bmjopen-2017017437).

Received 24 April 2017 Revised 13 June 2017 Accepted 21 June 2017

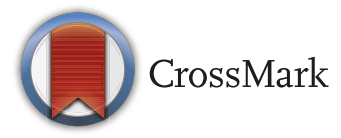

${ }^{1}$ Department of Nursing, Debre Berhan University, Debre Berhan, Ethiopia

${ }^{2}$ Department of Epidemiology and Rob Giel Research Centre, University Medical Centre Groningen, University of Groningen, Groningen, The Netherlands

${ }^{3}$ Department of Community Nutrition, Tehran University of Medical Sciences, Tehran, The Islamic Republic of Iran ${ }^{4}$ Department of Epidemiology, University Medical Centre Groningen, University of Groningen, Groningen, The Netherlands

Correspondence to Mr. Tesfa Dejenie Habtewold; tesfadej2003@gmail.com

\section{ABSTRACT}

Introduction Infant and young child feeding (IYCF) is the cornerstone of infant and child survival, healthy growth and development, healthy future generations and national development. In spite of the importance of optimal nutrition in low- and middle-income countries, there has been no review conducted in Ethiopia. Thus, the aim of this systematic review and meta-analysis is to estimate the national coverage and identify the associated factors of IYCF practices in Ethiopia.

Methods PubMed, Scopus, EMBASE, CINHAL, EBSCO, Web of Science and WHO Global Health Library databases will be searched for all available publications from 1 January 2000 to 30 September 2017. All published studies on the timely initiation of breast feeding, exclusive breast feeding and timely initiation of complementary feeding practice in Ethiopia will be screened, selected and reviewed. Bibliographies of identified articles and grey literature will be hand-searched as well. Heterogeneity of studies will be quantified using Higgins's method where $\mathrm{I}^{2}$ statistic $>80 \%$ indicates substantial heterogeneity. Funnel plots and Egger's regression test will be used to assess potential publication bias. The Newcastle-0ttawa Scale (NOS) will be used to assess the quality of evidence and risk of bias. Meta-analysis and meta-regression will be carried out to estimate the pooled national prevalence rate and an OR of each associated factor of IYCF practices. Narrative synthesis will be performed if meta-analysis is not feasible due to the substantial heterogeneity of studies. Ethics and dissemination Ethical clearance is not required for this study because primary data will not be collected. The results of this systematic review and metaanalysis will be published in a peer-reviewed journal and presented at an (inter)national research symposium. Systematic review registration This systematic review and meta-analysis has been registered with the International Prospective Register of Systematic Reviews (PROSPERO), registration number CRD42017056768.

\section{INTRODUCTION}

The United Nations (UN) Convention on Child Rights declared that every infant and child has the right to good nutrition. ${ }^{1}$ Infant and young child feeding (IYCF) is the cornerstone of infant and child survival, healthy

\section{Strengths and limitations of this study}

- The latest published data will be reviewed and meta-analysed.

- The first meta-analysed national evidence on infant and young child feeding practices (IYCF) will be provided.

- This review and meta-analysis will be conducted in line with international guidelines and statements.

- A multilevel theoretical model for primary care intervention and research will be developed.

- The new evidence may not be representative of other nations and/or continents because only studies conducted in Ethiopia will be included.

Inferences on causality may be difficult.

growth and development, a healthy future generation and national development. ${ }^{2}$ Breast feeding is a natural practice by all mothers and has numerous benefits for the infant, mother and society at large. ${ }^{3}$ It reduces the risk of infection and increases intelligence and educational attainment of the child; it also prevents maternal overweight, diabetes and cancer. $^{2}{ }^{3}$ In addition, breast feeding maintains skin-to-skin contact that facilitates safe colonisation of the newborn skin by the mother's skin bacteria and prevents hypothermia. ${ }^{4}$ Each year breast feeding saves the life of about 823000 children and 20000 mothers, and 302 billion dollars. ${ }^{35}$ In low-and middle-income countries, recent data show that optimal breast feeding prevents about $12 \%$ of under-5 child mortality every year. ${ }^{6}$ Other contemporary studies in Ethiopia, Ghana, Bolivia and Madagascar have shown that breast feeding prevents $20-22 \%$ of neonatal deaths. ${ }^{7-9}$

Suboptimal IYCF practices increase the risk of infant and child morbidity and mortality by up to fivefold. ${ }^{10-12}$ Child malnutrition causes approximately 2.7 million deaths per year, 156 million stunting, 50 million wasting 
and 42 million overweight or obesity. ${ }^{13}$ In developing countries, approximately 25-50\% of infant mortality is attributed to suboptimal IYCF practices. $^{11}{ }^{14}$ The Central Statistical Agency (CSA) 2011 report shows that the neonatal mortality rate accounts for $42 \%$ of under- 5 mortality in Ethiopia. ${ }^{15}$ Due to the heterogeneous nature of barriers, ${ }^{4617}$ achieving optimum IYCF practices is the major challenge in developing and developed countries. ${ }^{18}$ To improve, maintain and promote IYCF practices, the World Health Organization (WHO) defined eight core indicators and seven optional indicators. ${ }^{19}$ This review will focus only on three core indicators: timely initiation of breast feeding (TIBF), exclusive breast feeding and timely initiation of complementary feeding (TICF). The rationale for choosing these indicators is that (1) studies have been published frequently on these indicators compared with others; and (2) these indicators address the feeding practice of newborn infants under 1-year-old, which is believed to be the critical period of growth and development. ${ }^{20}$

TIBF is the percentage of children born in the last 2 years who were breastfed within the first hour of birth. ${ }^{19}$ The prevalence of TIBF in Eastern and Southern Africa is $59 \%$ compared with a global prevalence of $39 \% .{ }^{1421} \mathrm{~A}$ systematic review of 18 studies conducted in Asia, Africa and South America showed that the prevalence of TIBF ranges from $11.4 \%$ to $83.3 \%{ }^{4}$ A recent national survey report from 53 WHO European region countries showed that the prevalence of TIBF ranges from $5 \%$ to $84 \% .{ }^{22}$ According to the WHO rating on IYCF practices, the prevalence of $0-29 \%$ is poor, $30-49 \%$ is fair, $50-89 \%$ is good and $90-100 \%$ is very good. ${ }^{23}$ TIBF has been associated with the place of residence and delivery, ${ }^{24}$ educational status and postnatal advice on breast feeding, ${ }^{8}$ unemployment benefit and social welfare, ${ }^{25}$ maternal age and socioeconomic status, ${ }^{26}$ marital status and breast feeding exposure,${ }^{27}$ parity, ${ }^{28}$ antenatal care follow-up ${ }^{29}$ and postnatal care follow-up. ${ }^{8}$

Exclusive breast feeding is defined as the proportion of infants who exclusively breast fed during the first $0-5$ months after birth. ${ }^{30}$ The prevalence of exclusive breast feeding in Eastern and Southern Africa is $42 \%$ compared with a global prevalence of $37 \% .^{14}$ The prevalence of exclusive breast feeding in 53 WHO European region countries ranges from $21 \%$ to $30 \% .{ }^{22}$ Furthermore, the prevalence of exclusive breast feeding is $38-62 \%$ in India, ${ }^{31}{ }^{32} 42-44 \%$ in $\operatorname{Iran}^{33}$ and $53 \%$ in Guatemala. ${ }^{24}$ In accordance with the WHO rating on IYCF practices, a prevalence of $0-11 \%$ is poor, $12-49 \%$ is fair, $50-89 \%$ is good and $90-100 \%$ is very good. ${ }^{23}$ Exclusive breast feeding has been associated with the number of children, maternal and child age, educational and marital status, wealth index, antenatal and postnatal care follow-up, place of delivery, sex of newborn, birth order, family income, parents' education and employment, mode of delivery and TIBF. ${ }^{84} 29$ 33-36 On the other hand, a prospective study conducted in India showed that there has been no significant association between exclusive breast feeding and parental education, living conditions, antenatal care follow-up, birth weight, culture, postnatal breast feeding advice, previous breast feeding exposure and mothers' employment. ${ }^{31}$

TICF is defined as the proportion of infants aged 6-8 months who started additional solid or semi-solid or soft foods along with breast milk. ${ }^{19}$ The prevalence of TICF is $63.6 \%$ in Egypt, ${ }^{37} 57 \%$ in Nigeria, ${ }^{38}$ $39.2 \%$ in Pakistan, ${ }^{39} 84 \%$ in Sri Lanka ${ }^{40}$ and $55 \%$ in India. ${ }^{41}$ Furthermore, a Danish study showed TICF in $87 \%$ of infants. ${ }^{42}$ According to the WHO rating on IYCF practices, a prevalence of $0-59 \%$ is poor, $60-79 \%$ is fair, $80-94 \%$ is good and $95-100 \%$ is very good. ${ }^{23}$ TICF has been associated with antenatal and postnatal care follow-up, ${ }^{39}$ household income, ${ }^{41}$ educational and employment status, ${ }^{434}$ maternal and child age ${ }^{45}$ parental confidence, ${ }^{46}$ limited access to mass media, ${ }^{45}$ and religion, exclusive breast feeding and no siblings. ${ }^{47}$

In Ethiopia, the prevalence of TIBF ranges from $41.6 \%$ to $83.7 \%,{ }^{4}{ }^{48}$ exclusive breast feeding ranges from $13 \%$ to $79 \%^{49-51}$ and TICF ranges from $42 \%$ to $63 \% .^{52-55}$ According to WHO rating, TIBF and exclusive breast feeding coverage in Ethiopia are good whereas TICF is fair. ${ }^{23}$ The most common identified associated factors are sex of newborn, educational status, occupational status, parity, antenatal and postnatal care follow-up and birth preparedness. ${ }^{52-55}$

To optimise national IYCF practices, the Ethiopian government has been training health professionals, developing and revising procedural manuals and implementing the community integrated management of childhood illnesses and Baby-friendly Hospital Initiative programme based on expert opinion, international research and adapted tools. ${ }^{56-58}$ However, these international researches, guidelines and recommendations may not be effective in bringing behavioural, attitudinal and skill changes among mothers living in the different communities. ${ }^{11} 18$

Several systematic reviews and meta-analyses have been conducted on TIBF, ${ }^{47}$ exclusive breast feeding, ${ }^{16}$ long-term effects of breast feeding, ${ }^{59}$ breast feeding and intelligence ${ }^{60}$ duration of exclusive breast feeding ${ }^{30}$ and effectiveness of complementary feeding. ${ }^{61}$ Given the population cross-sociocultural diversity and multidimensional nature of barriers of IYCF practices, these systematic reviews and meta-analyses do not provide all-inclusive evidence and none of them have provided national estimates specific to Ethiopia. Researches on IYCF practices have been appearing from different parts of Ethiopia and published frequently in peer-reviewed (inter)national journals. However, there is no systematic review and meta-analysis of IYCF practices.

Taken together, there is an urgent need for a systematic review and meta-analysis of studies conducted in Ethiopia. This is the first systematic review and meta-analysis of IYCF practices in Ethiopia. The aim of this study is to answer the following research questions: (1) What is/are the national prevalence and associated factors of TIBF? 
(2) What is/are the national prevalence and associated factors of exclusive breast feeding? (3) What is/are the national prevalence and associated factors of TICF?

This systematic review and meta-analysis will provide consolidated data on the prevalence and associated factors of IYCF practices that would be useful for decision making. Besides, a multilevel theoretical model will be developed based on the Ethiopian context that would be useful for primary care intervention and research. To build the model, all negatively associated factors of IYCF practices will first be identified. Second, using the conceptual framework from the previous meta-analysis, ${ }^{416}$ these identified factors will be operationalised and categorised into four levels. Level 1, called proximal factors which need immediate intervention, includes maternal work and maternity leave, mothers' or families' knowledge on breast feeding, difficulties in breast feeding, coexistence and family support, caregiver of child, child health, use of pacifier, maternal care satisfaction and guidance on breast feeding. Level 2, called proximal intermediate factors, includes place of delivery, maternity financing, breast feeding practice, intention to breast feed, mode of delivery, birth weight, gestational age and postpartum complication. Level 3, called distal intermediate factors, includes maternal nutrition status, desire for pregnancy, smoking, alcoholism, antenatal and prenatal visits and prenatal financing. Level 4, called distal factors, includes paternal education, household income, family size, breast feeding experience and marital status. Maternal age, sex of newborn and place of residence are non-modifiable or miscellaneous factors.

\section{METHODS AND ANALYSIS}

\section{Protocol registration and review reporting}

The present systematic review and meta-analysis has been registered in the International Prospective Register of Systematic Reviews (PROSPERO), University of York Centre for Reviews and Dissemination (http://www. crd.york.ac.uk/PROSPERO/display_record.asp? ID= CRD42017056768) on 12 April 2017. Preferred Reporting Items for Systematic Review and Meta-Analysis Protocols (PRISMA-P) 2015 statement ${ }^{62}$ is used to write this protocol (see online supplementary file 1 for a completed copy of the PRISMA-P checklist). Meta-analysis Of Observational Studies in Epidemiology (MOOSE) guideline ${ }^{63}$ and/or PRISMA statement guideline ${ }^{64} 65$ will be used to report the review results. The screening and selection process of the reviewed articles will be illustrated using a PRISMA flow diagram (see online supplementary file 2).

\section{Data source and search strategy}

PubMed, Scopus, EMBASE, CINHAL, EBSCO, Web of Science and WHO Global Health Library databases will be searched for all available publications. Bibliographies of identified articles and grey literature will be hand-searched as well. Missed data will be handled by contacting the corresponding author/s. Search terms and strings of TIBF and exclusive breast feeding are adapted from systematic review and meta-analysis studies by Esteves et $a l,{ }^{4}$ Sharma et $a l,{ }^{17}$ Boccolini $e t a l^{16}$ and Khan et al. ${ }^{66}$ Similarly, search terms and strings of TICF are adapted from studies by Dewey et $a l^{61}$ and Vissers $e t a l^{67}$ The core search terms and phrases are 'breast feeding', 'breast milk', 'infant feeding', 'child feeding', 'time factors', 'start', 'first hour' and 'Ethiopia'. A comprehensive search strategy has been developed using appropriate Boolean operators in consultation with a medical information specialist and will be adapted to the abovementioned databases. PubMed database searching strings and strategy are presented in online supplementary file 3 .

\section{Inclusion and exclusion criteria}

Cross-sectional, case-control and cohort studies will be included. In addition, regional or national survey reports will be included. Further inclusion criteria are: all studies should be conducted in Ethiopia, reports of the prevalence rate of IYCF practices based on the WHO IYCF definition ${ }^{19}$ and at least one 'least adjusted' associated factor. All studies published in English from 1 January 2000 to 30 September 2017 will be included. Studies on premature newborn infants, infants in neonatal intensive care unit or a special care baby unit, low birth weight and mothers or infants with medical problems will be excluded. Likewise, commentaries, anonymous reports, letters, duplicate studies, editorials, qualitative studies and citations without full text will be excluded.

\section{Study screening and selection}

First, all studies obtained from all databases will be exported to RefWorks version 2.0 software (http:// www.refworks.com) and close and exact duplicates will be removed. Second, all studies will be exported to Microsoft Excel spreadsheet. Third, two independent reviewers will screen the title and abstract of each study. Agreement between the two reviewers will be accepted if Cohen's kappa coefficient is $>0.600 .{ }^{68}$ The screening will be repeated if the kappa value is $<0.60$. Fourth, after reaching good agreement, a full-text review will be performed. Finally, two investigators will independently extract the name of the first author and year of publication, region, study area, study design, study population, sample size, data collection procedure, IYCF practice, prevalence, least adjusted associated factors and effect sizes (OR) with $95 \%$ CIs using a structured data abstraction form (see online supplementary file 4).

\section{Risk of bias and quality assessment}

The Newcastle-Ottawa Scale (NOS) will be used to assess the quality of evidence and risk of bias in cohort and case-control studies. ${ }^{69}$ For cross-sectional studies, the adapted version of NOS will be used. ${ }^{69}$ NOS has a good inter-rater reliability and validity. ${ }^{70}$ It includes three categorical criteria with a maximum score of 9: a maximum of four stars allotted for 'selection'; a maximum of two stars allotted for 'comparability'; and a maximum of three stars allotted for 'outcome'. The quality of each study will be rated using the following 
scoring algorithm: $\geq 7$, 'good'; 2-6, 'fair'; and $\leq 1$, 'poor' ${ }^{72}$ Only studies of 'good' quality will be selected for the final review and analysis. In addition, the strength of the evidence will be graded based on the three domains of quality, quantity and consistency ${ }^{73}$ The scoring of each item for each study will be summarised and presented using a table. Further, the quality assessment will be complemented by administering the Strengthening the Reporting of Observational Studies in Epidemiology (STROBE) statement. ${ }^{74}$ Any disagreements will be resolved by discussion between the two reviewers; otherwise, the third reviewer will decide.

\section{Measuring outcome variables}

TIBF is the percentage of newborns who breast fed within the first hour of birth. ${ }^{19}$ Exclusive breast feeding is defined as the proportion of infants who exclusively breast fed during $0-5$ months since birth. ${ }^{19}{ }^{30}$ TICF is defined as the proportion of infants who started additional foods and liquids along with breast milk between 6 and 8 months of age. $^{19}$

\section{Data synthesis, publication bias and statistical analysis}

The data will be synthesised based on the three selected IYCF practice core indicators. Funnel plots and Egger's regression test will be used to assess potential publication bias. ${ }^{75}$ Based on the power and the number of studies that will be included in the analysis, other tests of publication bias will also be considered. Before meta-analysis, arc-sine transformation of the prevalence estimates will be used to adjust the effect of high prevalence studies on the pooled estimate. ${ }^{76}$ Heterogeneity of studies will be quantified using the Higgins et $a l^{77}$ method where $\mathrm{I}^{2}$ statistic $>80 \%$ indicates substantial heterogeneity. In addition, heterogeneity will be assessed manually considering the study area and demographic characteristics of the study population. Meta-analysis and meta-regression will be carried out to calculate the pooled prevalence rate and an OR of each associated factor of TIBF, exclusive breast feeding and TICF separately. Effect size will be estimated using fixedand random-effect models and the model that provides a higher estimate will be reported. ${ }^{78}{ }^{79}$ In addition, a separate forest plot will be constructed. Narrative synthesis will be performed if meta-analysis is not feasible due to the substantial heterogeneity of studies. The data will be inputted and analysed using Review Manager version 5.3.5 software for Windows. ${ }^{80}$

\section{Subgroup analysis}

Subgroup analysis will be carried out based on the regional states and place of residence (urban versus rural) where the study is conducted as defined by the Federal Democratic Republic of Ethiopia.

\section{Ethics and dissemination}

Ethical clearance is not required for this review because primary data will not be collected. The results of this systematic review and meta-analysis will be published in a peer-reviewed journal and presented at an (inter) national research symposium.
Acknowledgements We are grateful to Sjoukje van der Werf, medical information specialist at the University of Groningen, for her valuable support in designing the search strategy.

Contributors TDH conceived and designed the study. TDH and BST developed the search strategy. TDH, M.Al, NTS, SHM, MMB and BST wrote the protocol. All the authors read the manuscript and have given the final approval for publication.

Funding This research received no specific grant from any funding agency in the public, commercial or not-for-profit sectors.

\section{Competing interests None declared.}

Ethics approval Ethical clearance is not required for this review because primary data will not be collected. The results of this systematic review and meta-analysis will be published in a peer-reviewed journal and presented in (inter)national research symposium.

Provenance and peer review Not commissioned; externally peer reviewed.

Open Access This is an Open Access article distributed in accordance with the Creative Commons Attribution Non Commercial (CC BY-NC 4.0) license, which permits others to distribute, remix, adapt, build upon this work non-commercially, and license their derivative works on different terms, provided the original work is properly cited and the use is non-commercial. See: http://creativecommons.org/ licenses/by-nc/4.0/

(c) Article author(s) (or their employer(s) unless otherwise stated in the text of the article) 2017. All rights reserved. No commercial use is permitted unless otherwise expressly granted.

\section{REFERENCE}

1. United Nations Office of the High Commissioner on the Rights of the Child. Conventions onthe rights of the child. http://www.ohchr.org/ EN/Professionallnterest/Pages/CRC.aspx. (accessed 11 April 2017).

2. Hansen K. Breastfeeding: a smart investment in people and in economies. Lancet 2016;387:416.

3. Anon. Breastfeeding: achieving the new normal. Lancet 2016;387:404.

4. Esteves TM, Daumas RP, de Oliveira MI, et al. Factors associated to breastfeeding in the first hour of life: systematic review. Rev Saude Publica 2014;48:697-708.

5. Rollins NC, Bhandari N, Hajeebhoy N, et al. Why invest, and what it will take to improve breastfeeding practices? Lancet 2016;387:491-504.

6. Black RE, Victora CG, Walker SP, et al. Maternal and child undernutrition and overweight in low-income and middle-income countries. Lancet 2013;382:427-51.

7. Edmond KM, Zandoh C, Quigley MA, et al. Delayed breastfeeding initiation increases risk of neonatal mortality. Pediatrics 2006;117:e38 $0-\mathrm{e} 386$.

8. Setegn T, Gerbaba M, Belachew T. Determinants of timely initiation of breastfeeding among mothers in Goba Woreda, South East Ethiopia: a cross sectional study. BMC Public Health 2011;11:1.

9. Baker EJ, Sanei LC, Franklin N. Early initiation of and exclusive breastfeeding in large-scale community-based programmes in Bolivia and Madagascar. J Health Popul Nutr 2006;24:530-9.

10. Elizabeth KE. Feeding of young infants and children in exceptionally difficult circumstances (like, HIV and humanitarian emergencies). Solution exchange MCH Community Newsletter Breastfeeding Month Special. 2008.

11. World Health Organization. Exclusive breastfeeding for six months best for babies everywhere, 2011. 2012.

12. Ogbo FA, Page A, Idoko J, et al. Diarrhoea and suboptimal feeding practices in Nigeria: evidence from the National Household surveys. Paediatr Perinat Epidemiol 2016;30:346-55.

13. World Health Organization. Infant and young child feeding. http:// www.who.int/mediacentre/factsheets/fs342/en. (accessed $11 \mathrm{Apr}$ 2017).

14. Begum K, Dewey KG. Impact of early initiation of exclusive breastfeeding on newborndeaths. 2010.

15. Central Statistical Agency and ICF International. Ethiopia demographic and health survey 2011. Addis Ababa, Ethiopia and Calverton, Maryland, USA, 2012.

16. Boccolini CS, Carvalho ML, Oliveira MI. Factors associated with exclusive breastfeeding in the first six months of life in Brazil: a systematic review. Rev Saude Publica 2015;49:pii.

17. Sharma IK, Byrne A. Early initiation of breastfeeding: a systematic literature review of factors and barriers in South Asia. Int Breastfeed J 2016;11:1. 
18. UNICEF. Nutrition: Breastfeeding and complementary feeding. 2015. http://www.unicef.org/nutrition/index_breastfeeding.html. (accessed 10 Jun 2016)

19. World Health Organization. Indicators for assessing infant and young child feeding practices: Part 1: definitions. Conclusions of a consensus meeting held 6-8 November 2007. Washington DC, USA, 2008.

20. Nurliyana AR, Mohd Shariff Z, Mohd Taib MN, et al. Early nutrition, growth and cognitive development of infants from birth to 2 years in Malaysia: a study protocol. BMC Pediatr 2016;16:160.

21. Victora CG, Bahl R, Barros AJ, et al. Breastfeeding in the 21st century: epidemiology, mechanisms, and lifelong effect. Lancet 2016;387:475-90.

22. Bagci Bosi AT, Eriksen KG, Sobko T, et al. Breastfeeding practices and policies in WHO European region member states. Public Health Nutr 2016;19:753-64.

23. World Health Organization. Infant and young child feeding: a tool for assessing national practices policies and programmes. 2003.

24. Dearden K, Altaye M, De Maza I, et al. Determinants of optimal breast-feeding in peri-urban Guatemala City, Guatemala. Rev Panam Salud Publica 2002;12:185-92.

25. Flacking R, Nyqvist $\mathrm{KH}$, Ewald U. Effects of socioeconomic status on breastfeeding duration in mothers of preterm and term infants. Eur $J$ Public Health 2007;17:579-84.

26. Morhason-Bello IO, Adedokun BO, Ojengbede OA. Social support during childbirth as a catalyst for early breastfeeding initiation for first-time Nigerian mothers. Int Breastfeed J 2009;4:16.

27. Tarrant RC, Kearney JM. Session 1: Public health nutrition. Breastfeeding practices in Ireland. Proc Nutr Soc 2008;67:371-80.

28. Horii N, Guyon AB, Quinn VJ. Determinants of delayed initiation of breastfeeding in rural Ethiopia: programmatic implications. Food Nutr Bull 2011;32:94-102.

29. Dhandapany G, Bethou A, Arunagirinathan A, et al. Antenatal counseling on breastfeeding -- is it adequate? A descriptive study from Pondicherry, India. Int Breastfeed J 2008;3:5.

30. Kramer MS, Kakuma R. The optimal duration of exclusive breastfeeding. Protecting infants through human milk. Springer, 2004:63-77.

31. Chudasama RK, Amin CD, Parikh YN. Prevalence of exclusive breastfeeding and its determinants in first 6 months of life: a prospective study. Int J Health Allied Sci 2009;8.

32. Madhu K, Chowdary S, Masthi R. Breast feeding practices and newborn care in rural areas: a descriptive cross-sectional study. Indian J Community Med 2009;34:243-6.

33. Koosha A, Hashemifesharaki R, Mousavinasab N. Breast-feeding patterns and factors determining exclusive breast-feeding. Singapore Med J 2008;49:1002.

34. Uchendu U, Ikefuna A, Emodi I. Factors associated with exclusive breastfeeding among mothers seen at the University of Nigeria Teaching Hospital. S Afr J Child Health 2009;3.

35. Alemayehu T, Haidar J, Habte D. Determinants of exclusive breastfeeding practices in Ethiopia. Ethiop J Health Sci 2009;23.

36. Hector D, King L, Webb K, et al. Factors affecting breastfeeding practices: applying a conceptual framework. NSW Public Health Bull 2005;16:52-5.

37. El Shafei AM, Labib JR. Determinants of exclusive breastfeeding and introduction of complementary foods in rural Egyptian communities. Glob J Health Sci 2014;6:236-44.

38. Ogbo FA, Page A, Idoko J, et al. Trends in complementary feeding indicators in Nigeria, 2003-2013. BMJ Open 2015;5:e008467.

39. Hazir T, Senarath U, Agho K, et al. Determinants of inappropriate timing of introducing solid, semi-solid or soft food to infants in Pakistan: secondary data analysis of demographic and health survey 2006-2007. Matern Child Nutr 2012;8(Suppl 1):78-88

40. Senarath U, Godakandage SS, Jayawickrama H, et al. Determinants of inappropriate complementary feeding practices in young children in Sri Lanka: secondary data analysis of demographic and Health Survey 2006-2007. Matern Child Nutr 2012;8(Suppl 1):60-77.

41. Patel A, Pusdekar Y, Badhoniya N, et al. Determinants of inappropriate complementary feeding practices in young children in India: secondary analysis of National Family Health Survey 2005 2006. Matern Child Nutr 2012;8(Suppl 1):28-44

42. Kronborg H, Foverskov E, Væth M. Breastfeeding and introduction of complementary food in Danish infants. Scand J Public Health 2015;43:138-45.

43. Kabir I, Khanam M, Agho KE, et al. Determinants of inappropriate complementary feeding practices in infant and young children in Bangladesh: secondary data analysis of Demographic Health Survey 2007. Matern Child Nutr 2012;8(Suppl 1):11-27.

44. Joshi N, Agho KE, Dibley MJ, et al. Determinants of inappropriate complementary feeding practices in young children in Nepal: secondary data analysis of Demographic and Health Survey 2006. Matern Child Nutr 2012;8(Suppl 1):45-59.

45. Victor R, Baines SK, Agho KE, et al. Factors associated with inappropriate complementary feeding practices among children aged 6-23 months in Tanzania. Matern Child Nutr 2014;10:545-61.

46. Tatone-Tokuda F, Dubois L, Girard M. Psychosocial determinants of the early introduction of complementary foods. Health Educ Behav 2009;36:302-20.

47. Ogunlesi TA, Ayeni VA, Adekanmbi AF, et al. Determinants of timely initiation of complementary feeding among children aged 6-24 months in Sagamu, Nigeria. Niger J Clin Pract 2014;17:785-90.

48. Beyene MG, Geda NR, Habtewold TD, et al. Early initiation of breastfeeding among mothers of children under the age of 24 months in Southern Ethiopia. Int Breastfeed J 2016;12:1.

49. Asemahagn MA. Determinants of exclusive breastfeeding practices among mothers in Azezo district, northwest Ethiopia. Int Breastfeed J 2016;11:22

50. Hailu C. Assessment of knowledge. Attitude and practice among mothers about VCT and feeding of infants born to HIV positive women. Jimma Town, Ethiopia, 2005.

51. Hailu M, Gebremariam A, Alemseged F. Knowledge about obstetric danger signs among pregnant women in Aleta Wondo District, Sidama Zone, Southern Ethiopia. Ethiop J Health Sci 2010;20:2532.

52. Sisay W, Edris M, Tariku A. Determinants of timely initiation of complementary feeding among mothers with children aged 6-23 months in Lalibela District, Northeast Ethiopia, 2015. BMC Public Health 2016;16:884.

53. Haile D, Belachew T, Berhanu G, et al. Complementary feeding practices and associated factors among HIV positive mothers in Southern Ethiopia. J Health Popul Nutr 2015;34:1.

54. Semahegn A, Tesfaye G, Bogale A. Complementary feeding practice of mothers and associated factors in Hiwot Fana Specialized Hospital, Eastern Ethiopia. Pan Afr Med J 2014;18:143.

55. Shumey A, Demissie M, Berhane Y. Timely initiation of complementary feeding and associated factors among children aged 6 to 12 months in Northern Ethiopia: an institution-based crosssectional study. BMC Public Health 2013;13:1.

56. Ethiopia Federal Ministry of Health. Ethiopian national strategy for infant and young child feeding. 2004

57. Miller NP, Amouzou A, Tafesse M, et al. Integrated community case management of childhood illness in Ethiopia: implementation strength and quality of care. Am J Trop Med Hyg 2014;91:424-34.

58. Labbok MH. Global baby-friendly hospital initiative monitoring data: update and discussion. Breastfeed Med 2012;7:210-22.

59. Horta BL, Victora CG. Long-term effects of breastfeeding: a systematic review. 2013.

60. Horta BL, Loret de Mola C, Victora CG. Breastfeeding and intelligence: a systematic review and meta-analysis. Acta Paediatr 2015;104:14-19.

61. Dewey KG, Adu-Afarwuah S. Systematic review of the efficacy and effectiveness of complementary feeding interventions in developing countries. Matern Child Nutr 2008;4(Suppl 1):24-85.

62. Moher D, Shamseer L, Clarke M, et al. Preferred Reporting Items for Systematic Review and Meta-Analysis Protocols (PRISMA-P) 2015 statement. Syst Rev 2015;4:1

63. Stroup DF, Berlin JA, Morton SC, et al. Meta-analysis of observational studies in epidemiology: a proposal for reporting. JAMA 2000;283:2008-12.

64. Beller EM, Glasziou PP, Altman DG, et al. PRISMA for abstracts reporting systematic reviews in journal and conference abstracts. PLoS Med 2013;10:e1001419.

65. Moher D, Liberati A, Tetzlaff J, et al. Preferred reporting items for systematic reviews and meta-analyses: the PRISMA statement. PLoS Med 2009;6:e1000097.

66. Khan J, Vesel L, Bahl R, et al. Timing of breastfeeding initiation and exclusivity of breastfeeding during the first month of life: effects on neonatal mortality and morbidity--a systematic review and metaanalysis. Matern Child Health J 2015;19:468-79.

67. Vissers KM, Feskens EJ, van Goudoever JB, et al. The timing of complementary feeding in preterm infants and the effect on overweight: study protocol for a systematic review. Syst Rev 2016;5:149.

68. Viera AJ, Garrett JM. Understanding interobserver agreement: the kappa statistic. Fam Med 2005;37:360-3.

69. Peterson J, Welch V, Losos M, et al. The Newcastle-Ottawa scale (NOS) for assessing the quality of nonrandomised studies in metaanalyses. 2011.

70. Hartling L, Hamm M, Milne A, et al. Validity and inter-rater reliability testing of qualityassessment instruments [internet]. 2012. 12-EHC039-EF. 
71. Hootman JM, Driban JB, Sitler MR, et al. Reliability and validity of three quality rating instruments for systematic reviews of observational studies. Res Synth Methods 2011;2:110-8.

72. McPheeters ML, Kripalani S, Peterson NB, et al. Closing the quality gap: revisiting the state of the science (Vol 3: Quality improvement interventions to address health disparities). Evid Rep Technol Assess 2012;208.3:1-475

73. West S, King V, Carey TS, et al. Systems to rate the strength of scientific evidence. Evid Rep Technol Assess 2002:1-11.

74. von Elm E, Altman DG, Egger M, et al. The Strengthening the Reporting of Observational Studies in Epidemiology (STROBE) statement: guidelines for reporting observational studies. Prev Med 2007;45:247-51.
75. Egger M, Davey Smith G, Schneider M, et al. Bias in meta-analysis detected by a simple, graphical test. BMJ 1997;315:629-34.

76. Barendregt JJ, Doi SA, Lee YY, et al. Meta-analysis of prevalence. $J$ Epidemiol Community Health 2013:67:974-8.

77. Higgins JP, Thompson SG. Quantifying heterogeneity in a metaanalysis. Stat Med 2002;21:1539-58.

78. DerSimonian R, Laird N. Meta-analysis in clinical trials revisited. Contemp Clin Trials 2015;45:139-45.

79. Borenstein M, Hedges LV, Higgins JP, et al. A basic introduction to fixed-effect and random-effects models for meta-analysis. Res Synth Methods 2010;1:97-111.

80. The Cochrane Collaboration. Review manager (RevMan)(computer program) version 5.3. Cochrane Reviewers' Handbook 4.0. 2014. 